\title{
Neurological Complications of Myeloproliferative Syndromes with Negative Philadelphia Chromosome (MPS Ph-) in Lome Tertiary Hospital
}

\author{
Komi Assogba ${ }^{1, ~}$, Kodzo Vinyo Kumako ${ }^{1}$, Kossivi Martin Apetse ${ }^{1}$, Essohana Justin Padaro ${ }^{2}$, \\ Abago Balaka ${ }^{3}$, Abdoullaye Idrissou ${ }^{1}$, Komi Igneza Agbotsou ${ }^{1}$, Nyenèvi Komla Anayo, \\ Abdullah Blakime', Agnon Ayélola Koffi Balogou' \\ ${ }^{1}$ Neurology Service, Campus University Hospital, Lomé, Togo \\ ${ }^{2}$ Clinical Hematology Services, Campus University Hospital, Lomé, Togo \\ ${ }^{3}$ Internal Medicine Service, Sylvanus Olympio University Hospital, Lomé, Togo
}

Email address:

seraphinassogba@hotmail.com (K. Assogba)

${ }^{*}$ Corresponding author

\section{To cite this article:}

Komi Assogba, Kodzo Vinyo Kumako, Kossivi Martin Apetse, Essohana Justin Padaro, Abago Balaka, Abdoullaye Idrissou, Komi Igneza Agbotsou, Nyenèvi Komla Anayo, Abdullah Blakime, Agnon Ayélola Koffi Balogou. Neurological Complications of Myeloproliferative Syndromes with Negative Philadelphia Chromosome (MPS Ph-) in Lome Tertiary Hospital. Clinical Neurology and Neuroscience. Vol. 3, No. 1, 2019, pp. 11-15. doi: 10.11648/j.cnn.20190301.13

Received: February 13, 2019; Accepted: March 15, 2019; Published: May 27, 2019

\begin{abstract}
Introduction: Myeloproliferative syndromes with philadelphia (MPS Ph) chromosome negative are diseases little known in our environment and cause grave neurological sequels. The study aimed to describe the neurological complications of these syndromes. Patients and method: It was a retrospective cross-sectional study carried out on the files of patients follow up or hospitalized in hematology or neurology departments of our tertiary hospital from January, 2008 to December, 2017. The variables analyzed were composed of epidemiological data, clinical signs, treatments used, neurological complications, and evolution. Results: Among 39 patients with MPS Ph negative, 30 (76.9\%) had neurological complications at the time of diagnostic. Headaches, dizziness and splenomegaly were the most reported clinical signs in $95.2 \%, 73.6 \%$ and $66.7 \%$ respectively. Different types of MPS Ph negative were observed with 21 cases of polycythemia vera, 8 cases of essential thrombocythemia and one case of primary myelofibrosis. The research of Jack2V617F mutation was made in 25 patients (83.3\%) and was positive in 15 . The neurological complications were marked by peripheral neuropathy ( 20 cases), cerebral venous thrombosis (15 cases) and ischemic stroke in 11 cases. The average length of stay in hospital was 23.6 days. Concerning the treatment, $96.7 \%$ had received antiplatelet therapy and cytoreductive treatment was added in $66.7 \%$. The outcome was marked by the remission of symptoms in $11.1 \%$ of cases, $46.7 \%$ with sequels and $20 \%$ of death. Conclusion: The MPS $\mathrm{Ph}$ negative patients are often discovered in late stage of the disease progression with neurological complications. Measures need to be taken to improve the early diagnosis and management of MPS Ph chromosome negative.
\end{abstract}

Keywords: Myeloproliferative Syndrome, Neurological Complications, Philadelphia Chromosome Negative

\section{Introduction}

Developing countries are facing the demographic and epidemiological transition were non communicable diseases such as hematological diseases will replace infections and malnutrition [1]. Myeloproliferative syndromes (MPS) are characterized by the excessive production of mature myeloid blood cells of chronic evolution [2, 3]. MPSs are divided into conventional and atypical MPSs. Conventional MPS is divided in four distinct clinical entities including chronic myeloid leukemia (CML) with and without Philadelphia chromosome $(\mathrm{Ph})$, polycythemia vera $(\mathrm{PV})$, essential thrombocythaemia (ET), and primary myelofibrosis (PMF) $[4,5]$. Myeloproliferative syndromes cause many medical 
complications. Neurological disturbances, one of these complications, have been estimated between 12 and $80 \%$ [6, 7]. These complications are consecutive to blood hyperviscosity mechanisms. The clinical signs are marked by headaches, vertigo, abnormal choreic movements, and motor and sensory deficits [8-10]. This study had aimed to report different aspects of neurological complications due to MPS with Philadelphia negative chromosome (MPS Ph-).

\section{Methodology}

\subsection{Framework of Study}

The study had taken place in the hematology and neurology departments of the Lomé University Hospital. Both services have 50 beds with qualified staff, specialized units and equipment adapted to provide cares, teaching and research activities.

\subsection{Patients and Study Method}

It was a retrospective, cross-sectional and descriptive study covered a period from January, 2008 to December, 2017. The study dealt with the files of patients having neurological complications related to myeloproliferative syndromes with Philadelphia chromosome-negative. The diagnosis of myeloproliferative syndromes with negative Philadelphia chromosome was made on the WHO diagnostic criteria [4, 5]. The majority of patients were referred to the hematology department based on abnormal blood count cells obtained for various conditions. Neurological complications were reported either at diagnosis or during medical follow-up. Patients were followed up and benefited from quarterly appointments in both departments. Telephone calls or and trips were made to the home of some patients to complete their neurological and hematological examinations and to obtain their informed consent prior inclusion. The data were collected on preestablished survey from. The analyzed data were composed with epidemiological, clinical, therapeutic, evolutionary and biological (hemoglobin, hematocrit, platelet count, white cells count, sedimentation rate, and Jack2V617F mutation) variables. Cases of hematologic malignancies, secondary MPSs and MPS of doubt diagnosis were not included in the study. The collected data were analyzed using Microsoft XP software Epi Info version 6.0 and Excel 2007. The statistical analysis focused on the comparison of variables between the two sexes. The accepted level of significance was $5 \%$.

\section{Results}

A total of 39 patients were diagnosed with MPS Ph negative. Thirty patients had neurological complications, which had represented $76.9 \%$. The majority of neurological complications were discovered at the time of diagnosis in $76.7 \%$ (23 cases) and after 5 years of progression in $13.3 \%$ (4 cases). The average age was $47.5 \pm 13.3$ years with extremes of 20 and 73 years. The age group of 50-59 years old was the most represented with $26.7 \%$. The sex ratio $(21 \mathrm{M} / 9 \mathrm{~F})$ was
2.3. The table 1 summarized the neurological complications of the myeloproliferative syndrome and the age group.

Table 1. Distribution of patient according to the neurological complications and age group.

\begin{tabular}{llllll}
\hline & IS & HS & MC & CVT & PN \\
\hline$[20-29]$ & 0 & 0 & 0 & 0 & 2 \\
{$[30-39]$} & 1 & 0 & 0 & 1 & 4 \\
{$[40-49]$} & 2 & 1 & 0 & 3 & 5 \\
{$[50-59]$} & 4 & 0 & 1 & 6 & 8 \\
{$[60-69]$} & 1 & 1 & 0 & 3 & 2 \\
$\geq 70$ & 1 & 0 & 0 & 2 & 1 \\
Total & 9 & 2 & 1 & 15 & 22 \\
\hline
\end{tabular}

Legend: IS: Ischemic stroke; HS: Hemorrhage stroke; MC: Medullary compression; CVT: Cerebral Venous thrombosis; PN: Peripheral neuropathy.

Regarding the clinical symptoms, headaches, vertigo and splenomegaly were the most reported in different types of MPS Ph negative. Different types of MPSs Ph negative were reported with 21 cases of PV, 8 cases of ET and 1 case of PMF. The search for the Jack2V617F mutation was performed in 25 patients, or $83.3 \%$, and was positive in 15 patients. Neurologic complications were more observed in the range of $50-60 \%$ hematocrit, hemoglobin $18-20 \mathrm{~g} / \mathrm{l}$, white blood cells between 4-10,000 elements per milliliter, and platelet counts between 400,000 and 1200,000 per milliliter. There was no statistically significant difference between the clinical signs and the main parameters of the blood cells counts (hematocrit, hemoglobin, platelets and white blood cells). Cerebral computed tomography had shown multiple lacuna and massive ischemia of the deep Sylvian arterial territories in 9 patients, and intra-parenchymal hematomas in 2 patients $(6.7 \%)$. Cerebral MRI was performed in one patient and showed a T2 weighted hypersignal, confirming the vascular lesions obtained by the $\mathrm{CT}$ scan. The electroneurography had confirmed the peripheral neuropathy in 16 patients. The distribution of patients according to neurological complications and the type of MPS Ph negative was established in table 2.

Table 2. Distribution of patient according to the clinical signs and MPS Ph negative type.

\begin{tabular}{lllllll}
\hline & PV & \multicolumn{3}{c}{ ET } & \multicolumn{3}{c}{ PMF } \\
\cline { 2 - 7 } & N & \% & N & \% & N & \% \\
\hline Headaches & 20 & 95.2 & 6 & 66.7 & 1 & 100 \\
Paresthesia* & 36 & 97.6 & 11 & 72.8 & 3 & 100 \\
Dizziness & 18 & 85.7 & 5 & 55.6 & 1 & 100 \\
Pruritus & 10 & 47.6 & 0 & 0 & 0 & 0 \\
Bulky abdomen & 16 & 76.2 & 4 & 44.4 & 1 & 100 \\
Movements disorders & 6 & 28.6 & 0 & 0 & 0 & 0 \\
Visual disturbances & 14 & 66.7 & 1 & 11.1 & 1 & 100 \\
Redness of teguments & 17 & 81 & 0 & 0 & 0 & 0 \\
Convulsions & 6 & 28.6 & 4 & 44.4 & 1 & 100 \\
Splenomegaly & 15 & 71.4 & 5 & 55.6 & 1 & 100 \\
Hepatomegaly & 8 & 38.1 & 3 & 33.3 & 1 & 100 \\
Motor deficit & 9 & 42.9 & 2 & 22.2 & 0 & 0 \\
Erythrosis & 17 & 81 & 0 & 0 & 0 & 0 \\
\hline
\end{tabular}

Legend: Paresthesia: Heaviness of legs, tingling, burning sensation; N: Number; \%: Percentage; MPS: Myeloproliferative syndrome; PV: Polycythemia Vera; ET: Esential Thrombocythemia; PMF: Primary Myelofibrosis 
The distribution of patients according to neurological complications and the type of MPS Ph negative was noted in the table 3 .

Table 3. Distribution of patient according to neurological complications and the type of MPS ph negative.

\begin{tabular}{lllllll}
\hline & PV & \multicolumn{3}{c}{ ET } & \multicolumn{3}{c}{ PMF } \\
\cline { 2 - 7 } & $\mathbf{N}$ & $\mathbf{\%}$ & $\mathbf{N}$ & $\mathbf{\%}$ & $\mathbf{N}$ & $\mathbf{\%}$ \\
\hline Ischemic stroke & 5 & 6.7 & 3 & 10 & 1 & 3.3 \\
Hemorrhagic stroke & 1 & 3.3 & 1 & 3.3 & 0 & 0 \\
Medullary compression & 1 & 3.3 & 0 & 0 & 0 & 0 \\
CVT & 8 & 26.7 & 6 & 20 & 1 & 3.3 \\
Peripheral neuropathy & 15 & 50 & 4 & 13.3 & 1 & 3.3 \\
\hline
\end{tabular}

Legend: PV: Polycythemia Vera; ET: Essential Thrombocythemia; PMF: Primary Myelofibrosis; MPS: Myeloproliferative syndrome; N: Number; \%: Percentage; CVT: Cerebral Venous Thrombosis

The average length of stay was 23.6 days with extremes of
1 and 28 days. The cytoreductive treatment was added to aspirin in $66.7 \%$ of cases with remission of symptoms in $11.1 \%$ of cases.

The table 4 had reported the distribution of patients by treatment and the type of MPS Ph negative.

Table 4. Distribution of patients according to treatment and the type of MPS $\mathrm{Ph}$ -

\begin{tabular}{lllllll}
\hline & PV & \multicolumn{3}{c}{ ET } & PMF \\
\cline { 2 - 7 } & $\mathbf{N}$ & $\mathbf{\%}$ & $\mathbf{N}$ & $\mathbf{\%}$ & $\mathbf{N}$ & $\mathbf{\%}$ \\
\hline Bloodletting & 21 & 70 & 0 & 0 & 0 & 0 \\
Hydroxyurea & 11 & 36.7 & 8 & 26.7 & 1 & 3.3 \\
Aspirin & 21 & 70 & 8 & 26.7 & 0 & 0 \\
\hline
\end{tabular}

Legend: PV: Polycythemia Vera; ET: Esential Thrombocythemia; PMF: Primitive Myelofibrosis; MPS: Myeloproliferative syndrome

The table 5 had reported the distribution of patients according to treatment and neurological complications.

Table 5. Distribution of patients according to treatment and neurological complications.

\begin{tabular}{|c|c|c|c|c|c|c|}
\hline & \multicolumn{2}{|c|}{ Bloodletting } & \multicolumn{2}{|c|}{ Hydroxyurea } & \multicolumn{2}{|c|}{ Aspirin } \\
\hline & $\mathbf{N}$ & $\%$ & $\mathbf{N}$ & $\%$ & $\mathbf{N}$ & $\%$ \\
\hline Ischemic stroke & 7 & 23.3 & 3 & 10 & 9 & 30 \\
\hline Hemorrhagic stroke & 1 & 3.3 & 1 & 3.3 & 0 & 0 \\
\hline Medullary compression & 1 & 3.3 & 0 & 0 & 0 & 0 \\
\hline Cerebral venous thrombosis & 15 & 50 & 9 & 30 & 15 & 50 \\
\hline Peripheral neuropathy & 13 & 43.3 & 4 & 13.3 & 19 & 63.3 \\
\hline
\end{tabular}

Legend: N: Number; \%: Percentage

The overall medical care was provided in $43.6 \%$ by the patient himself, in $46.2 \%$ by the family and in $10.3 \%$ by the insurance companies as reported in figure 1 .

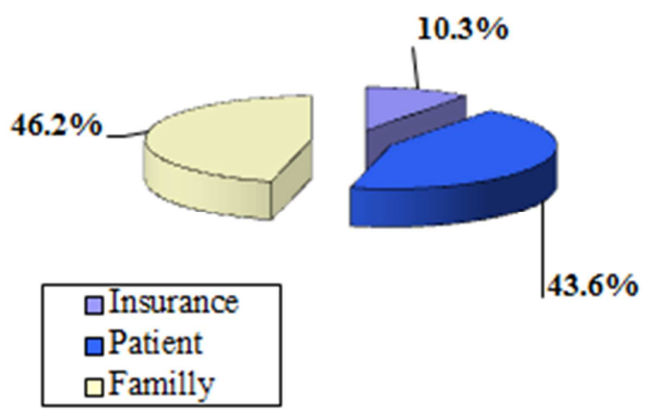

Figure 1. Distribution of patient according the type of care providers.

The evolution of complications was favorable in $26.7 \%$ of patients and the mortality rate was $20 \%$ as indicated in figure 2.

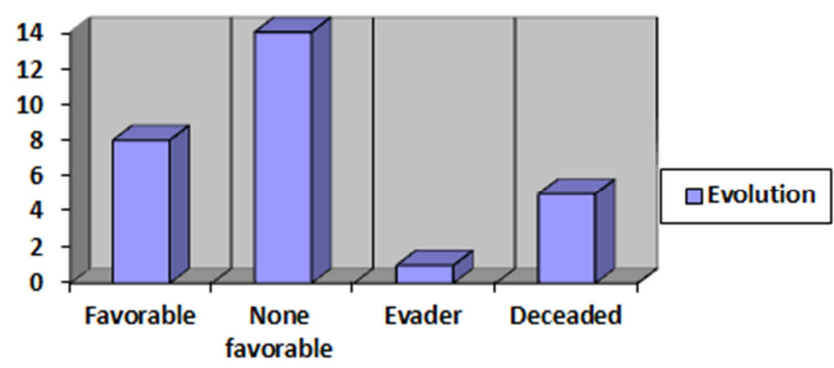

Figure 2. Distribution of patients according to the clinical evolution.

\section{Discussion}

The study reported ten-year follow-up of MPS Ph negative patients. In our daily working conditions in the tropics, this follow-up often remains irregular to maintain by the patients and the family. This is often the result of financial difficulties and rural population exodus related to socio-political conflicts or natural disasters. It is also a one center study and would not be widespread at a national scale. Nevertheless, the handicap of the neurological complications and the relevance of the obtained results deserve their share to the scientific community.

On epidemiological aspects, the study found that $76.9 \%$ of patients with MPSs $\mathrm{Ph}$ negative had neurological complications. Of the patients with ET, eight had neurological complications, which accounted for $66.7 \%$. The highest rate of neurological complications was observed in patients aged from 50 to 60 years $(26.7 \%)$. This result is higher than that observed in previous studies which reported neurological symptoms around $25.7 \%$ to $29.7 \%$ of patients followed up for ET $[9,10]$. We have noted that the rates found in these past studies were very low compared to ours. The lack of systematic reviews performed in patients during regular consultations and the delayed of diagnosis, could explain the high rate of complications at the late stage of the disease progression. Age and sex did not have a significant influence on the occurrence of neurological complications in patients, $p>0.08$. Some authors have reported superior results to ours related to youth study population $[11,12]$.

Neurological complications were discovered at the time of 
MPS Ph diagnostics or during follow-up in $76.9 \%$ of cases, much higher than the reported rate of $25.7 \%$ in some previous studies $[9,13,14]$. This high rate is explained once again by the technical platform insufficiencies of the myeloproliferative syndromes diagnostic and the ignorance of symptoms by the populations. Headaches, vertigo and splenomegaly were the majority signs observed in patients with MPSs. Similar results were reported in previous studies $[10,15,16]$.

The most observed grave neurological complications have been represented by cerebral venous thrombosis and ischemic strokes, similar results were reported in the literature $[11,17$, 18]. There was no significant correlation between hemoglobin, hematocrit, white blood cell count, and platelet count with the frequency of neurological symptoms, $\mathrm{p}>0.06$.

The search of Jack 2 V617F mutation was performed in 25 patients and positive in 15 . Patients with positive Jack2V617F mutation have high risk to develop neurological pathologies related to MPS Ph chromosome negative [18-20]. The high costs of these genetic examinations, the lack of adherence to health insurance and the low socioeconomic income of the majority of our patients may explain this low rate of achievement of biological checkup.

With regard to the treatment, the majority of patients have received antiplatelet therapy and cytoreductors. Cytoreductive treatment is often unavailable and difficult to access because of its high cost. Marrow bone transplantation and other genetic therapies were also impossible to access [21-23]. Medical care was supported either by the patient himself or by his family, and only $10.2 \%$ had health insurance. In most cases, it is the patient's entourage who bored the costs of hospital cares in situations of financial difficulties in the African regions. Despite this antiplatelet and cytoreductive treatment, the neurological sequels were heavy and the survival rate is low $[23,24]$. It is essential to consider other easy access therapeutic approaches and available for the most vulnerable populations. There is also great importance to underline the benefits of early diagnosis and management of patients with myeloproliferative syndromes $[25,26]$.

\section{Conclusion}

The study had reported patients with neurological complications of myeloproliferative syndrome with Philadelphia chromosome negative. Cerebral venous thrombosis and ischemic strokes were the major neurological complications observed at the time of diagnostic. Treatment was based on aspirin and cytoreductive therapy. Research, identification, and early care of patients with MPS Ph (-) will reduce neurological complications and improve quality of life. An easy access to new available therapies with universal public help insurance is the main tools to fill the social gaps.

\section{Conflicts of Interest}

The authors declare that they have no competing interests.

\section{References}

[1] Johansson P, Kutti J, Andreasson B, Safai-Kutti S, Vilen L, Wedel $\mathrm{H}$ et al. Trends in the incidence of chronic Philadelphia chromosome negative ( $\mathrm{Ph}-$ ) myeloproliferative disorders in the city of Goteborg, Sweden, during 1983-99. J Intern Med. 2004; 256 (2): 161-5.

[2] Finazzi G. Incidence and risk factors for bleeding in 1104 patients with essential thrombocythemia or prefibrotic myelofibrosis diagnosed according to the 2008 WHO criteria. Leukemia. 2012; 26 (4): 716-9.

[3] Gianelli U, Bossi A, Cortinovis I. Reproducibility of the WHO histological criteria for the diagnosis of Philadelphia chromosome-negative myeloproliferative neoplasms. Mod Pathol 2014; 27: 814-22.

[4] Moulard O, Mehta J, Fryzek J et al. Epidemiology of myelofibrosis, essential thrombocythemia, and polycythemia vera in the European Union. Eur J Haematol 2014; 92: 289-97.

[5] Marchioli R. Cardiovascular events and intensity of treatment in polycythemia vera. N Engl J Med. 2013; 368 (1): 22-33.

[6] Marchioli R, Finazzi G, Landolfi R, Kutti J, Gisslinger H, Patrono $\mathrm{C}$ et al. Vascular and neoplastic risk in a large cohort of patients with polycythemia vera. J Clin Oncol. 2005; 23 (10): 2224-32.

[7] Titmarsh GJ, Duncombe AS, McMullin MF et al. How common are myeloproliferative neoplasms? A systematic review and meta-analysis. Am J Hematol 2014; 89: 581-7.

[8] Billot S, Kouroupi EG, Le Guilloux J, Cassinat B, Jardin C, Laperche Th, et al. Neurological disorders in essential thrombocythemia. Haematologica 2011; 96 (12): 1866-9.

[9] Campbell PJ, Green AR. The myeloproliferative disorders. $N$ Engl J Med. 2006; 355: 2452-66.

[10] Vannucchi AM. Philadelphia chromosome-negative chronic myeloproliferative neoplasms: ESMO Clinical Practice Guidelines for diagnosis, treatment and follow-up. Ann Oncol. 2015; 26 (5): v85-99.

[11] Nangalia J, Grinfeld J, Green AR. Pathogenesis of Myeloproliferative Disorders. Annu Rev Pathol. 2016; 11: 101-26.

[12] Thoennissen NH, Koeffler HPh. Leukaemic Transformation of Philadelphia-chromosome-negative Myeloproliferative Neoplasms - A Review of the Molecular Background. European Oncology \& Haematology, 2011; 7 (1): 59-62.

[13] Elliott MA, Tefferi A. Thrombosis and haemorrhage in polycythaemia vera and essential thrombocythaemia. $B r J$ Haematol. 2005; 128 (3): 275-90.

[14] Viny AD, Levine RL. Genetics of myeloproliferative neoplasms. Cancer J. 2014; 20 (1): 61-5.

[15] Wu Zh, Zhang X, Xu X, Chen Y, Hu T, Kang Zh, et al. The mutation profile of JAK2 and CALR in Chinese Han patients with Philadelphia chromosome-negative myeloproliferative neoplasms. J Hematol Oncol 2014; 7: 48-59.

[16] Benton ChB, Tanaka M, Wilson C, Pierce Sh, Zhou L, Cortes $\mathrm{J}$, et al. Increased likelihood of post-polycythemia vera myelofibrosis in Ph-negative MPN patients with chromosome 12 abnormalities. Leuk Res. 2015; 39 (4): 419-23. 
[17] Spivak J. Narrative review on Thrombocytosis, polycythemia vera, and JAK2 mutations: The phenotypic mimiking of chronic myeloproliferation. Ann Intern Med. 2010; 152 (5): 300-6.

[18] Tefferi A, Vaidya R, Caramazza D, Finke C, Lasho T, Pardanani A. Circulating interleukin (IL)-8, IL-2R, IL-12, and IL-15 levels are independently prognostic in primary myelofibrosis: a comprehensive cytokine profiling study. J Clin Oncol. 2011; 29 (10): 1356-63.

[19] Alvarez-Larran A. Risk of thrombosis according to need of phlebotomies in patients with polycythemia vera treated with hydroxyurea. Haematologica. 2017; 102 (1): 103-9.

[20] Thiele J, Kvasnicka HM, Müllauer L. Essential thrombocythemia versus early primary myelofibrosis: a multicenter study to validate the WHO classification. Blood 2011; 117: 5710-8.

[21] Vainchenker W, Leroy E, Gilles L, Marty C, Plo I, Stefan N. Constantinescu. JAK inhibitors for the treatment of myeloproliferative neoplasms and other disorders Research 2018; 7: 82-100.
[22] Beauverd Y. Pegylated interferon alpha-2a for essential thrombocythemia during pregnancy: outcome and safety. A case serie. Haematologica. 2016; 101 (5): 182-4.

[23] Boddu P, Falchi L, Hosing Ch, Newberry K, Bose P, Verstovsek Sr. The role of thrombocytapheresis in the contemporary management of hyperthrombocytosis in myeloproliferative neoplasms: a case-based review. Leuk Res. 2017; 58: 14-22.

[24] Birgegard G. The Use of Anagrelide in Myeloproliferative Neoplasms, with Focus on Essential Thrombocythemia. Curr Hematol Malig Rep. 2016; 11 (5): 348-55.

[25] Cervantes F, Dupriez B, Passamonti F. Improving survival trends in primary myelofibrosis: an international study. J Clin Oncol 2012; 30: 2981-7.

[26] Amy Zh, Amber A, Stephen T. Prognostication in Philadelphia Chromosome Negative Myeloproliferative Neoplasms: A review of the recent literature. Curr Hematol Malig Rep. 2017; 12 (5): 397-405. 